\title{
El tiempo infantil de consumo televisivo y su relación con otras actividades
}

\author{
Javier Callejo \\ Profesor Titular de Sociología. UNED
}

\section{Resumen:}

A partir de los resultados de la última Encuesta de Empleo del Tiempo, realizada por el Instituto Nacional de Estadística, este trabajo analiza el lugar que tiene la relación con la televisión por parte de la audiencia infantil dentro del conjunto de actividades de estos a lo largo de un día medio y de distintos tipos de días. Asimismo se establece qué parte del tiempo de relación con la televisión la llevan a cabo en solitario. De manera previa, se establece una reflexión metodológica sobre las ventajas y limitaciones del uso de esta fuente de datos. Los resultados del estudio permiten señalar algunas de las consecuencias temporales y de estructuración del día aparejadas a lo que puede considerarse un excesivo consumo de televisión, como, también, relativizar algunas de las alarmas surgidas cuando se aborda este tema.

Palabras clave:

Consumo infantil de televisión, uso del tiempo, hábitos televisivos.

Abstract:

From the results of the last Survey of Employment of the Time, carried out by the National Institute of Statistics, this work analyzes the place that has the relation with the television on the part of the childlike audience inside the assembly of activities of these along a medium day and of different types of day. Likewise what part of the time of relation of children with the television they carry out it alone. In a prior way, a methodological reflection on the advantages is esta- 
blished and limitations of the use of this source of data. The results of the study permit to indicate some of the temporary consequences and of structuring of the day prepared to what can be considered an excessive consumption of television, as, also, to relativize some of the alarms arisen when this theme is undertaken.

Key words:

Children's televisión viewing, use of time, television habits.

\section{Introducción}

La relación infantil con la televisión ha sido fuente de preocupación de las sociedades desde que se empezó a comprobar la rápida introducción del medio de comunicación en los hogares. Puede decirse que ha sido una relación bajo constante sospecha, generando debates que han ascendido frecuentemente a la esfera política. Una preocupación que se dirige tanto a los mensajes que el niño recibe o potencialmente puede recibir desde la pantalla, como a la creciente duración diaria de la propia relación con el medio. El primer tipo de preocupación se ha proyectado en diversas normativas ${ }^{1}$, acuerdos de autorregulación ${ }^{2} \mathrm{y}$, allí donde existen, riguroso seguimiento de la programación en horario infantil de las instituciones de control, función apropiada en nuestro país por los diversos consejos autonómicos audiovisuales. El segundo tipo parece haberse dejado bajo la responsabilidad privada. Su corrección queda en el ámbito doméstico-familiar.

Tal preocupación colectiva en los países desarrollados ha generado una investigación que puede considerarse referencial para el conjunto de los campos. Baste destacar aquí las investigaciones empíricas del mundo anglosajón dirigidas respectivamente por Himmelweit ${ }^{3}$ y Schramm ${ }^{4}$, cuando la representación dominante del medio, en su relación con los niños, era

Como la Directiva europea Televisión sin Fronteras, de octubre de 1993.

2 En España, se encuentra ahora vigente el suscrito por las televisiones generalistas en diciembre de 2004, que tiene su escasamente productivo antecedente en el de abril de 1993.

3 Cfr. HIMMELWEIT, H., OPPENHEIM, A., y VINCE, P., Television and the child: An empricial study of the effects of television on the young, Londres, Oxford University Press, 1958.

4 Cfr. SCHRAMM, W., LYLE, J., y PARKER, E. B., Televisión para los niños, Barcelona, Hispano Europea, 1965. Esta in- 
menos negativa que la existente en la actualidad. Incluso, los adultos consultados subrayaban las capacidades formativas de la televisión, al mismo tiempo que se reconocía la evitación de los programas educativos por parte de los niños. Es decir, en momentos en que se podía decir de los adultos que aún eran conscientemente cómplices del seguimiento televisivo de los menores. Hoy, casi medio siglo después de convivencia televisiva, se da, al menos, lo que Núñez Ladevéze y Pérez Ornia han denominado disonancia pragmática ${ }^{5}$ para reflejar la distancia entre una dominante (mala) opinión sobre el medio y, por otro lado, una práctica de relación infantil con el medio que sigue creciendo. Es decir, una distancia entre los criterios normativos y la conducta que se lleva a cabo en relación con la televisión.

El trabajo que aquí se desarrolla es una aproximación a la conducta de relación de los niños con la televisión. Una relación observada sobre la dimensión tiempo, considerada como estratégica desde tres puntos de vista. En primer lugar, puesto que esta dimensión se establece: "como verdadero problema de la modernidad" ${ }^{6}$. En segundo lugar, porque, precisamente por el lugar principal que tiene el tiempo en nuestras vidas, permite observar el valor que se le da al consumo televisivo. Al fin y al cabo, el consumo de tiempo frente a la televisión es, también, consumo de vida. Especialmente como se hace aquí, poniendo el tiempo de relación con la televisión en comparación y articulación con el tiempo de otras actividades. En último lugar, admitido el tiempo como un recurso limitado, la dimensión temporal de la relación infantil con la televisión permite analizar los efectos de su variabilidad -más o menos tiempo de relación con el medio- sobre la dimensión temporal de las otras actividades, como estudiar o dormir; así como sobre otras actividades relacionadas con los medios de comunicación, como es el uso de Internet.

vestigación es considerada, sin duda, el punto de partida para quien desea conducir estudios en este campo, como señala FARNÉ, R., "Il problema della recerca su bambini e televisione”, ponencia del I Congreso Internacional la Televisión y la Infancia, Madrid, 17-19 de octubre de 2007.

5 NÚÑEZ LADEVĖZE, L., y PÉREZ ORNIA, J. R., "Los gustos de la audiencia infantil y la producción televisiva. El conflicto pragmático de los responsables de la audiencia infantil”, en Revista Española de Investigaciones Sociológicas, 99, 2002, pp. 113-143.

6 LUHMANN, N., Observaciones de la modernidad. Racionalidad y contingencia en la sociedad moderna, Barcelona, Paidós, 1997, p. 131. 
A pesar de la relevancia de la dimensión temporal, es escasa la presencia que tiene en la investigación española sobre niños y televisión (apartado 2). Tras exponer las capacidades y limitaciones del concepto de tiempo utilizado (apartado 3) y de la metodología de la que se ha servido la fuente de datos utilizados (apartado 4), se entra en los resultados del análisis, poniendo de relieve algunas características de ese tiempo de relación infantil con la televisión y sus consecuencias sobre el tiempo dedicado a otras actividades, así como si se trata de un tiempo en solitario o en compañía (apartado 5).

\section{La televisión y los niños}

Incluso cuando otros medios de comunicación parecen empezar a ser más atractivos que la propia televisión ${ }^{7}$, los estudios sobre la relación entre infancia y televisión constituyen una de las líneas de investigación centrales en las ciencias de la comunicación. Es más, parece que tienden a articularse los estudios de la relación de la infancia con la televisión y con Internet ${ }^{8}$ bajo la común preocupación colectiva de las consecuencias sobre el desarrollo de la infancia.

La relación con la televisión por parte de los niños ha preocupado desde el inicio del funcionamiento del medio. Así, se ha obtenido una acumulación de resultados de investigación, en múltiples direcciones, que, sin embargo apenas parecen haber tenido repercusión alguna sobre el quehacer productores y programadores televisivos o sobre la práctica cotidiana de la sociedad, tanto receptora última de las emisiones televisivas como de los resultados de tanta investigación. Ni la realidad televisiva, ni la de la vida de la infancia, parecen afectadas por tal cantidad de resultados.

\footnotetext{
7 Cfr. LIVINGSTONE, S., y BOBER, M., UK Children Go Online: Final Report of Key Project Findings, London School of Economics and Political Science, Londres, 2005; disponible en: www.children-go-online.net. El atractivo del mundo digital por parte de las nuevas audiencias queda expuesto en LIVINGSTONE, S., "The camping nature of audiences: from the Mass Audience to the interactive media user", en Valdivia, A (ed.), The Blacwell Companion to Media Research, Blackwell, Oxford, 2003, pp. 337-59.

8 Cfr. NÚÑEZ LADEVÉZE, L., y TORRECILLA LACAVE, L., “El contexto de la recepción infantil”, en Telos, nº. 73, octubrediciembre 2007, pp. 46-51.
} 
Una producción de resultados que empieza en España poco más allá de un lustro de funcionamiento del medio, con la encuesta que el padre Jesús María Vázquez analiza tras ser aplicada a los suscriptores de la revista Mundo Cristiano, más centrada en las opiniones y representaciones que la audiencia tenía de la relación entre televisión y niños que en referencia más directa a comportamientos. Algo que se va a mantener en buena parte de las encuestas, con cuestionario estandarizado, específicas sobre la relación con el medio, tanto por parte de los niños, como del conjunto de la población.

A los primeros pasos de investigación empírica sobre la relación entre la televisión y los niños, siguió una ancha línea de ensayos de variada calidad ${ }^{9}$, en los que se vuelve a hacer más hincapié en llamar la atención en clave de alarma social, que en la exposición de la conducta de los propios niños con relación al medio.

Dejando a un lado la extensa nómina de estudios centrados en análisis de contenido de los programas infantiles, aquí interesa resaltar las investigaciones que han ido en busca de los efectos de la televisión sobre los niños. Buena parte de ellos, enfocados en los efectos sobre las prácticas escolares ${ }^{10}$. Otra buena parte, en las consecuencias de los contenidos violentos y agresivos $^{11}$. Últimamente, los estudios recalcan el papel de los contextos concretos en los que los niños españoles establecen la relación con el medio de comunicación y, en especial, se

9 Cabe citar, como muestra de esta línea, los trabajos de RODRÍGUEZ MÉNDEZ, J. M., Los teleadictos, Barcelona, Laia, 1973; ALONSO, M., MATILLA, L., VÁZQUEZ, M., Los teleniños, Barcelona, Laia, 1986; ALONSO, M., MATILLA, L., VÁZQUEZ, M., Teleniños públicos, teleniños privados, Madrid, Quirón, 1995; VALLEJO-NÁJERA, A., Mi hijo ya no juega, sólo ve la televisión, Madrid, Temas de Hoy, 1987; FERRÉS, J., Educar en una cultura del espectáculo, Barcelona, Paidós, Barcelona, 2003.

${ }^{10}$ SÁNCHEZ HORCAJO, J. J., "Televisión y familia: Comunicación familiar, pasividad y rendimiento escolar”, en Sociedad y Utopía, nº.1 y 2, 1993, pp. 59-83 y 41-58 respectivamente; YOUNIS HERNÁNDEZ, J. A., El niño y la cultura audiovisual, Santa Cruz de Tenerife, Centro de la Cultura Popular Canaria, 1988; YOUNIS HERNÁNDEZ, J. A., El aula fuera del aula. La educación invisible de la cultura audiovisual, Las Palmas de G. C., Nogal, 1993.

${ }^{11}$ LEÓN, J. M., CANTERO, F. J., GÓMEZ, T., "Efectos de la clasificación del comportamiento del modelo simbólico televisado sobre la agresividad infantil”, Revista de Psicología Social, 12 (1), 1997, pp. 31-42; PEÑA, M. E., ANDREU, J. M., MUÑOZ, M. J., "Efectos de la visión de escenas violentas en la conducta agresiva infantil, Psicothema, vol. 11 (1), 1999 , pp. 27-36; y, sobre todo, el estudio de GARCÍA GALERA, M. C., Televisión, violencia e infancia. El impacto de los medios, Barcelona, Gedisa, 2000. 
destaca el papel mediador de la familia ${ }^{12}$. Es decir, se han producido resultados poniendo la investigación tanto en la pantalla (análisis de contenido), como en distintos aspectos de la relación infantil con el medio. Sin embargo, son pocos los que se toma la dimensión en tiempo. El estudio de Vera toma periféricamente el tiempo de consumo televisivo infantil, a partir de

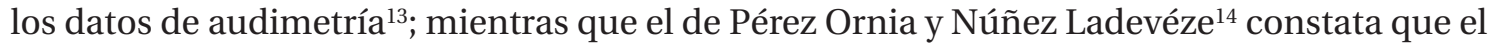
grupo de niños entre 4 y 12 años es el que menos tiempo de televisión consume y, además, que la mayor parte de ese tiempo se dedica a la programación dirigida a los adultos. Sin embargo, ningún estudio relevante ha enfocado el tiempo de consumo televisivo infantil en relación con otros tiempos, destinados a otras actividades.

En otros ámbitos, se ha enfocado directamente el tiempo televisivo de los niños en relación con otros tiempos. En el caso de la investigación de Mutz y otros ${ }^{15}$ para poner entre paréntesis las hipótesis que señalan una relación causal entre la cantidad de tiempo dedicada al consumo televisivo y las cantidades de tiempo dedicadas a otras actividades tenidas como relevantes. De especial interés y excelente diseño metodológico longitudinal, por lo que puede considerarse uno de los trabajos clásicos en el tema, es la investigación de Burton y otros ${ }^{16}$, donde el tiempo de consumo televisivo en edades tempranas es considerado la variable independiente sobre comportamientos en edades posteriores. El tiempo de consumo televisivo adquiere un lugar central en el diseño metodológico, aun cuando relativamente aislado del tiempo dedicado a otras actividades.

\footnotetext{
12 Cabe destacar al respecto, las aportaciones de VILCHEZ MARTIN, L. F., Televisión y familia, Un reto educativo, Madrid, PPC, 1999; NÚÑEZ LADEVĖZE, L., y PÉREZ ORNIA, J. R., op. cit.; y LLOPIS GOIG, R., "La mediación familiar del consumo infantil de televisión. Un análisis referido a la sociedad española”, Comunicación y Sociedad, vol XVII (2), 2004, pp. 125-147.

${ }^{13}$ VERA, M. J., “¿Cómo perciben los niños la televisión?”, en AA. VV., Televisión, niños y jóvenes, Valencia, RTVV, 1994, pp. 37-63.

${ }^{14}$ NÚÑEZ LADEVĖZE, L., PÉREZ ORNIA, J. R. "Programación infantil en la televisión española. Inadecuada relación entre oferta y demanda”, Telos, no. 54, enero-marzo, 2003, pp. 103-115.

${ }^{15}$ Cfr. MUTZ, D. C., ROBERTS, D. F., VAN VUUREN, D. P., “Reconsidering the displacement hypothesis: Television's influence on children's time use”, Communication Research, nº.20, 1993, pp. 51-75.

${ }^{16}$ Cfr. BURTON, J., CALONICO, J., MCSUENY, D., “Growing up with televisión: Effects of preschool televisión watching on first-grade children”, Journal of Communication, no. 29, 1979, pp. 164-170
} 


\section{Del tiempo televisivo al hábito televisivo}

Buena parte de la investigación sobre infancia y televisión ha consistido en un reto metodológico, que parte de la gran dificultad de su objeto de estudio. En primer lugar, la fácil tendencia a la mediación del prejuicio, ya que este se encuentra legitimada en defensa de una parte de tal objeto, los niños. Se genera así más un discurso normativo ${ }^{17}$, que un análisis científico.

Por otro lado, hay que reconocer los importantes problemas metodológicos que derivan del trabajo empírico con niños. Hay que tener en cuenta que, mientras la mayor parte de las prácticas de investigación social exigen la mediación del discurso, buena parte de la infancia se define (in-fans, el que no habla) por la falta de capacidad discursiva. Hay que tener en cuenta que casi todo el repertorio de aproximación social empírica está diseñado para la observación de adultos ${ }^{18}$, abriéndose la reflexión sobre la capacidad de simples adaptaciones a otros sujetos, como propone Rodríguez Pascual ${ }^{19}$.

Dificultades en la producción de material empírico que, además, se extienden a las posibilidades de generalización de los resultados de los que hay que considerar diseños de investigación central en este campo. Tanto los diseños de corte experimental, casi siempre con el horizonte puesto en la búsqueda de efectos sobre los niños de la relación televisiva, aun cuando quedándose en diseños cuasiexperimentales ${ }^{20}$ en el entorno escolar; como los

${ }^{17}$ El dominio de tal discurso normativo ha estado principalmente del lado de los análisis que han puesto la dimensión educativa como horizonte en la relación infantil con el medio. Véase, entre los autores españoles, PÉREZ TORNERO, J. M., El desafío educativo de la televisión. Para comprender y usar el medio, Barcelona, Paidós, 1994; GARCÍA MATILLA, A., Una televisión para la educación. La utopía posible, Barcelona, Gedisa, 2003; FERRÉS, J., op. cit.; o MARTA LAZO, C., La televisión en la mirada de los niños, Madrid, Fragua, 2005.

${ }^{18}$ CALLEJO, J., "Limitaciones para el establecimiento de una tipología de la investigación sobre niños y televisión: el caso español”, en Empiria. Revista de Metodología de Ciencias Sociales, no. 8, julio-diciembre 2004, p. 24.

${ }^{19}$ Cfr. RODRÍGUEZ PASCUAL, I., "Redefiniendo el trabajo metodológico cualitativo con niños: el uso de la entrevista de grupo aplicada al estudio de la tecnología”, en Empiria. Revista de Metodología de Ciencias Sociales, nº. 12, julio-diciembre 2006.

${ }^{20}$ Siguiendo la ya clásica división de categorías metodológicas entre diseños experimentales y diseños cuasiexperimentales o imperfectos, aun cuando potencialmente útiles si tienen sentido. División que debemos a CAMPBELL, D., y STANLEY, J., Diseños experimentales y cuasiexperimentales en la investigación social, Buenos Aires, Amorrortu, 1991. 
diseños centrados en la relación infantil con un único contenido televisivo (programa o género televisivo), presentan grandes dudas a la hora de la generalización de sus resultados ${ }^{21}$.

Ante tales dificultades, hay que reconocer como humildes y legítimos refugios tanto el estudio, a través de análisis de contenido, de los mensajes explícitamente destinados a la infancia, como la recientemente extendida mirada etnográfica sobre este campo, con una más o menos explícita renuncia a la generalización ${ }^{22}$. Sin embargo y a pesar de las evidentes dificultades, parece poco plausible que las ciencias sociales de la comunicación renunciasen a una observación empírica, con potencial de generalización, de la relación de la infancia con la televisión. Aquí se propone restablecerla sobre una dimensión básica, pero esencial, de la relación con la televisión, como es el tiempo de su consumo. La experiencia televisiva infantil -con sus consecuencias- es, antes que nada, una experiencia temporal, aunque, claro está, no sólo es esto.

La relación temporal con la televisión es uno de los principales resultados de la investigación comercial mediante el uso de audímetros, que potencialmente podrían pasar de ese mundo comercial de repartos de las audiencias y certificación del mercado publicitario televisivo ${ }^{23}$ a otros ámbitos. De hecho, hay que reconocer que ofrece una importante radiografía de los hábitos televisivos de la sociedad en general y de los niños en particular, entendiendo por tales la continuada repetición de conductas de relación con el medio. Algo que facilita su diseño como panel. Una concepción restringida del hábito, ya que sociológicamente es proyectado -y de ahí su papel estratégico como dispositivo de observación del conjunto de la sociedadcomo producto manifiesto de las condiciones de vida, como conducta diaria de la vida en unas condiciones de vida, tan inserto e identificador de la sociedad que llega a hablarse de hábito-habitus hereditario de una comunidad o sociedad ${ }^{24}$.

${ }^{21}$ GARCÍA MATILLA, A., CALLEJO GALLEGO, J., y WALZER, A., Los niños y los jóvenes frente a las pantallas, Madrid, Ministerio de Trabajo y Asuntos Sociales, 2004, pp. 38-39.

${ }^{22}$ Aun cuando en el centro de los debates metodológicos, una parte importante de la doctrina metodológica asume la incapacidad de generalización de la investigación cualitativa. Véase CORBETTA, P., Metodología y técnicas de investigación social, Madrid, McGraw-Hill, 2003, p.51.

${ }^{23}$ CALLEJO, J., Investigar las audiencias, Barcelona, Paidós, 2001.

${ }^{24}$ Véase principalmente en WEBER, M., Economía y Sociedad, México, Fondo de Cultura Económica, 1979, p. 320. 
Así, el hábito, además de compromiso en el tiempo proyectado en la repetición de conductas es principalmente compromiso con el tiempo o, lo que puede considerarse lo mismo, una actividad definida en una estructura de actividades. Siguiendo a Bourdieu y su concepto de habitus, más allá de las regularidades hay que destacar su capacidad de generar y reproducir regularidades, lo que obliga a ver de cada práctica sus constricciones, limitaciones y, sobre todo, su articulación con otras prácticas, es decir, la armonización y orquestación entre actividades, conformando las mismas un sistema ${ }^{25}$. Pues bien, la orquestación de las actividades a través del tiempo, en cuanto recurso limitado que restringe las posibilidades de articulación entre las mismas, parece un camino adecuado para llegar al hábito.

Desde la perspectiva señalada, el tiempo de relación con la televisión es más, aunque también, que el tiempo de consumo televisivo. Es el tiempo de relación con la televisión en relación con otras actividades, lo que nos aproxima al sentido que tiene para los sujetos ese tiempo y, en definitiva, a una concepción societal ${ }^{26}$ de ese tiempo televisivo de los niños, que contemple la integración de este tipo de tiempo, por su actividad central, en el conjunto de tiempos.

La integración social del tiempo de la relación con la televisión tiene dos principales vías de acceso. Desde la perspectiva del sentido dado por los individuos a ese tiempo, lo que tiende a recogerse preferentemente a partir de diseños de investigación con técnicas cualitativas productoras de discursos sobre las acciones, especialmente entrevistas abiertas. La segunda perspectiva parte de una concepción global y sistémica del tiempo, como un recurso del que los individuos disponen, siendo consciente de su limitación, para ser aplicado a distintas actividades. De esta manera, cada categoría social o cada sujeto presenta un sistema de actividades, articuladas de alguna manera entre sí, de forma que se asume, en función de su extensión relativa, la importancia que tiene cada una. Una y otra suponen reducciones ${ }^{27} \mathrm{ex}^{-}$

\footnotetext{
${ }^{25}$ BOURDIEU, P., El sentido práctico, Madrid, Taurus, 1991, p. 91 y stes.

${ }^{26}$ Esta propuesta de concepción societal del tiempo, aplicada al tiempo de trabajo, se encuentra en PRIETO, C., y RAMOS, R., "El tiempo de trabajo entre la competitividad y los tiempos sociales", en Las relaciones de empleo en España, F. Miguélez y Carlos Prieto (coords). Madrid, Siglo XXI, 1999.

${ }^{27}$ En el sentido que emplea este concepto LUHMANN, N., La Ciencia de la Sociedad, México, Anthropos, 1996, p. 261 y stes.
} 
cluyentes, capaces de observarse respectivamente ${ }^{28}$. Aquí sólo se aborda la segunda perspectiva, centrándonos en la distribución del tiempo de relación con la televisión entre distintas categorías de actividades.

\section{Metodología}

Los estudios sobre el uso del tiempo se han asentado en la mayor parte de los países desarrollados, con un horizonte internacional claramente destinado a la comparación entre unos países y otros $^{29}$. Tal interés por el estudio de cómo las sociedades usan el tiempo deriva principalmente de su consideración como un recurso, relativamente igual para todas las personas y sociedades; pero que es susceptible de ser aplicado y distribuido de muy distinta manera según cada sociedad. Visto así, el uso del tiempo se convierte en una especie de privilegiado indicador de la sociedad, capaz de condensarla en buena medida: habla de la sociedad. Usando una socorrida metáfora: el uso del tiempo se configura como retrato de la sociedad.

Ha de reconocerse que la mayor parte del esfuerzo institucional por saber el uso del tiempo en las sociedades se ha concentrado en la perspectiva cuantitativa, mediante encuesta con cuestionario estandarizado, llegándose a cierta homogeneización de las técnicas de estudio de empleo del tiempo ${ }^{30}$. Así se ha configurado un instrumento que ha adquirido nombre propio en el ámbito de los métodos y las técnicas de investigación social, como son las encuestas de presupuestos temporales. Desde el dominio de tal perspectiva cuantitativa, y, por lo tanto, de la medición, se ha hecho especial hincapié en la duración: en el tiempo manifestado de duración de las distintas actividades que los sujetos realizan a lo largo de un día. Cada momento del día tiene su actividad. Cada actividad, un tiempo en clave de duración. Hay que hacer notar que tal operacionalización de los tiempos sociales no deja de ser una reducción, como ocurre en todo proceso de operacionalización ${ }^{31}$, de ese concepto complejo que es el

\footnotetext{
${ }^{28}$ Como puede derivarse de las propuestas de LUHMANN, N., Observaciones de la modernidad..., op. cit..

${ }^{29}$ Un horizonte institucionalizado en asociaciones como la Internacional Association for Time Use Research (IATUR) o la European Foundation for the Improvement of Living and Working Conditions.

${ }^{30}$ SZALAI, A. (ed.), The use of time, The Hague, Mouton, 1972.

${ }^{31}$ Cfr. CORBETTA, P., op. cit., p. 82 y stes.
} 
tiempo. Así, se hacen todos los momentos iguales, todas las duraciones idénticas, sin tener en cuenta los aspectos cualitativos de los distintos momentos y, por lo tanto, las vivencias temporales o sentido que los individuos dan a tales duraciones.

El trabajo que aquí se presenta constituye una aproximación analítica a los datos sobre el uso del tiempo que ofrece el último estudio nacional en clave de presupuestos temporales: la Encuesta de Empleo del Tiempo 2002-2003, desarrollada por el Instituto Nacional de Estadística $^{32}$. Esta operación estadística está diseñada para obtener datos comparativos, a nivel de la Unión Europea, de la distribución y utilización del tiempo de las personas. Recaba información de una muestra de 20.603 hogares y 46.774 individuos de más de diez años, distribuida uniformemente a lo largo del año (entre octubre de 2002 y septiembre de 2003), con el objetivo de que se encuentren representados todos los días del año a escala de estrato y comunidad autónoma, aun cuando la submuestra del denominado fin de semana (viernes a domingo) tiene un peso relativamente mayor para poder observar la mayor variedad de comportamientos durante este período semanal.

La obtención de la información se realiza mediante:

a) Cuestionario del hogar, en el que se selecciona un informante. Su estructura es: la composición del hogar, atención a los menores de 10 años, disponibilidad de servicio doméstico, características y bienes de equipamiento de la vivienda principal, cultivo de plantas y cuidado de animales, ingresos económicos y ayudas recibidas por el hogar.

b) Cuestionario individual. Está especialmente centrado en las actividades del sujeto entrevistado: económicas, en el hogar, de voluntariado, ocio, etc. Salvo para el caso de la actividad laboral, el período de referencia sobre el que se cuestiona la realización de la actividad está compuesto por cuatro semanas.

c) Diario de empleo del tiempo, durante las 24 horas de un día, en intervalos de diez minutos. Abarca desde el intervalo entre las seis y las seis y diez de la mañana, hasta el intervalo entre las cinco y cuenta minutos y las seis de la mañana del día siguiente.

${ }^{32}$ Los microdatos del estudio pueden obtenerse en las páginas web del INE: www.ine.es 
Puede considerarse el registro central del estudio. En él, se pide al informante que lleve el diario a lo largo del día y lo rellene con regularidad, registrando, para cada intervalo de diez minutos, las actividades realizadas (primaria y secundaria o simultánea) y las características de la compañía con la que se realizan tales actividades

Para nuestro estudio, se ha segmentado la muestra total en una submuestra de niños entre 10 y 13 años, con un tamaño de 1.893 individuos: 907 de 10 y 11 años, y 986 de 12 y 13 años. Del total de la muestra nacional, corresponde a la observada apenas algo más del $4 \%$. Tamaño de la submuestra que ha de tenerse por suficiente para abordar algunas dimensiones de la realidad que pretendemos, siempre que el proceso de desagregación no sea excesivo.

Con la finalidad de evitar niveles de demasiada desagregación, con la consiguiente pérdida de significación estadística, se ha concentrado el conjunto de actividades en seis categorías, cuya sola denominación habla de sus contenidos: cuidados personales, tiempo de trabajo, tiempo de estudios, tiempo dedicado al hogar (tareas domésticas) y el cuidado de la familia, tiempo de ocio y actividades afines y, por último, tiempo empleado en trayectos. Categorías excluyentes y que presentan muy diverso grado de homogeneidad, en cuanto al supuesto sentido del tiempo dedicado a las distintas actividades que contiene. Así, mientras tal homogeneidad es más atribuible a los tiempos de trabajo, estudio, ocio o trayectos; lo es menos con respecto a la categoría de cuidados personales o la destinada a recoger el tiempo dedicado al hogar y la familia. La primera, incluye desde actividades para la cobertura de necesidades fisiológicas (dormir, aseo), que es la denominación que adquiere esta categoría en una buena parte de los estudios de presupuestos temporales, hasta otras con una intensa proyección social, como ocurre con la comida. Por su lado, la doble cara de la categoría de actividades dedicadas al hogar y la familia, queda ya inscrita en su propia denominación.

Hay que señalar que en el trabajo que aquí se presenta, de algunas de las categorías de actividades señaladas se ha extraído alguna actividad específica cuando el análisis lo ha requerido. Es lo que ocurre con la actividad de dormir que, dada la importancia que tiene en toda la población y en los niños en particular, se ha analizado aparte de la categoría de actividades que lo engloba, que es la de cuidados personales. Lo mismo ha ocurrido, en otro tipo de análisis que se verá en su momento, con la comida. 
Si la gran ventaja de esta operación estadística consiste en la potencial articulación de toda actividad con otras actividades bajo un único recurso y, por lo tanto, un único marco, el tiempo, sus principales limitaciones con respecto a nuestro objeto de estudio se desarrollan en dos direcciones. Por un lado, el relacionado con la forma de recoger la información mediante cuestionario autoadministrado sobre las actividades del día en el diario de empleo del tiempo, que es el que más nos interesa. Por lo tanto, el sujeto no es observado sino que refleja su autoobservación a un segundo observados mediante sus inscripciones en el cuestionario, mediando, por lo tanto, las apreciaciones del sujeto observado, como tiende ocurrir en toda encuesta mediante cuestionario estandarizado. En este caso, además, limitado en la edad de los participantes a un umbral en el que pudieran rellenar por ellos mismos el instrumento de recogida de la información. Tal umbral se ha situado en los diez años.

Por otro lado, la referencia de la recogida de información se establece sobre la actividad principal en tramos de diez minutos. Categoría de actividad principal que puede arrastrar connotaciones valorativas, dejando a un lado de las referencias de los encuestados actividades poco valoradas y de corta duración, por no mencionar las que tienden a quedar fuera de todo proceso de cuestionamiento, como son las actividades poco o nada socialmente legitimadas. En nuestra actividad de referencia, el consumo de televisión, tenderán a quedar fuera los fugaces vistazos a la pantalla en el momento que se estén realizando otras actividades: comer en un restaurante, por ejemplo. No obstante y para evitar en la medida de lo posible tal limitación del método de recogida de información, como principio metodológico general en este trabajo se ha tendido a sumar la relación con la televisión, tanto referida como actividad primaria, como referida como actividad secundaria o actividad que acompaña, haciéndose simultáneamente, a una primaria, en ese mismo tramo de diez minutos.

A pesar de tales limitaciones, hay que subrayar el enorme potencial de los resultados de esta operación estadística para el análisis de los más diversos campos sociológicos. En España, hay que resaltar el fructífero uso de la misma que han hecho Ramos ${ }^{33}$, para el análisis com-

${ }^{33}$ Cfr. RAMOS, R., Cronos dividido, Madrid, Centro de Investigaciones Sociológicas, 1990. 
parativo entre sociedades, y Durán ${ }^{34}$, utilizándola como base para el establecimiento de la aportación del trabajo doméstico al Producto Interior Bruto, de estar contabilizada tal aportación por las cuentas públicas. Esta es la primera vez que, en nuestro país, se proyecta su uso en el análisis de la relación con los medios de comunicación.

\section{Resultados}

\subsection{Dimensiones iniciales del tiempo infantil frente a la televisión}

El tiempo medio diario de seguimiento televisivo como actividad principal es de 123,77 minutos entre los niños de 10 y 11 años y de 129,88 minutos entre los niños de 12 y 13 años. El 40,25\% del conjunto de estos niños, también señala el seguimiento televisivo como actividad secundaria en algún momento del día. Es más, un 12,6\% lo señala en tres momentos del día. Si sumamos la televisión como actividad principal y como actividad secundaria, el total de niños que en algún momento del día ve al menos diez minutos de televisión es del 92,1\%. La media de tiempo consumido, entre los que ven televisión, es de 149,55 minutos/día.

Aproximadamente, dos horas y medias de media de consumo total (como actividad primaria y secundaria) infantil televisivo diario. Algo que puede ser ciertamente preocupante; pero tal dimensión queda relativizada al compararla con otros grupos de edad. La media de minutos/día de los niños (149,55 minutos/día) está bastante por debajo de la media general: 171,79 minutos/día (más de 20 minutos de diferencia). La media de tiempo mayor se encuentra entre los mayores de 65 años, entre los que alcanza los 234,95 minutos/día, seguida de los que tienen entre 41 y 65 años, con 167,67 minutos/día. Los que menos televisión, como actividad principal, consumen, son los que se encuentran entre 25 y 40 años, con 139,26 minutos/día.

El día de la semana que mayor porcentaje de niños consume al menos algo de televisión es el martes. El que menos, el lunes:

${ }^{34}$ Cfr. DURÁN, M. A., La cuenta satélite del trabajo no remunerado en la Comunidad de Madrid, Madrid, Comunidad de Madrid, 2006. 
Tabla número 1: porcentaje de niños que vieron televisión y tiempo de consumo (minutos/día) por día de la semana

\begin{tabular}{lcc}
\hline Día semana & porcentaje vió tv & Minutos/día entre los que vieron tv \\
\hline Lunes & 89,11 & 124,52 \\
Martes & 93,47 & 138,38 \\
Miércoles & 90,91 & 124,27 \\
Jueves & 91,77 & 135,99 \\
Viernes & 93,42 & 151,54 \\
Sábado & 93,02 & 180,29 \\
Domingo & 92,07 & 175,41 \\
\hline
\end{tabular}

Sin embargo, el día en que más se ve televisión, por aquellos que la ven, es el sábado, sobrepasando las tres horas; mientras que el miércoles es el día que menos se ve televisión por aquellos que la ven, sobrepasando apenas las dos horas: 124,27 minutos día. Este ciclo semanal coincide sólo parcialmente con el del resto de categorías de edad y, lo más relevante, difiere del ciclo semanal de consumo televisivo de los adolescentes (entre 14 y 17 años), que tiende a un consumo menos pronunciado, en comparación con la población total, durante el fin de semana. Para el total de la población, es el domingo el día de mayor seguimiento televisivo, entre los que lo siguen; mientras que para los adolescentes y, en mayor grado, para los niños es el sábado. Así, durante este día del fin de semana, los niños ven, de media, casi una hora más de televisión que en días como el lunes o el miércoles. 
Conico I: Thaps nedis do consums to por da de la kemana, tegon grupse so edad

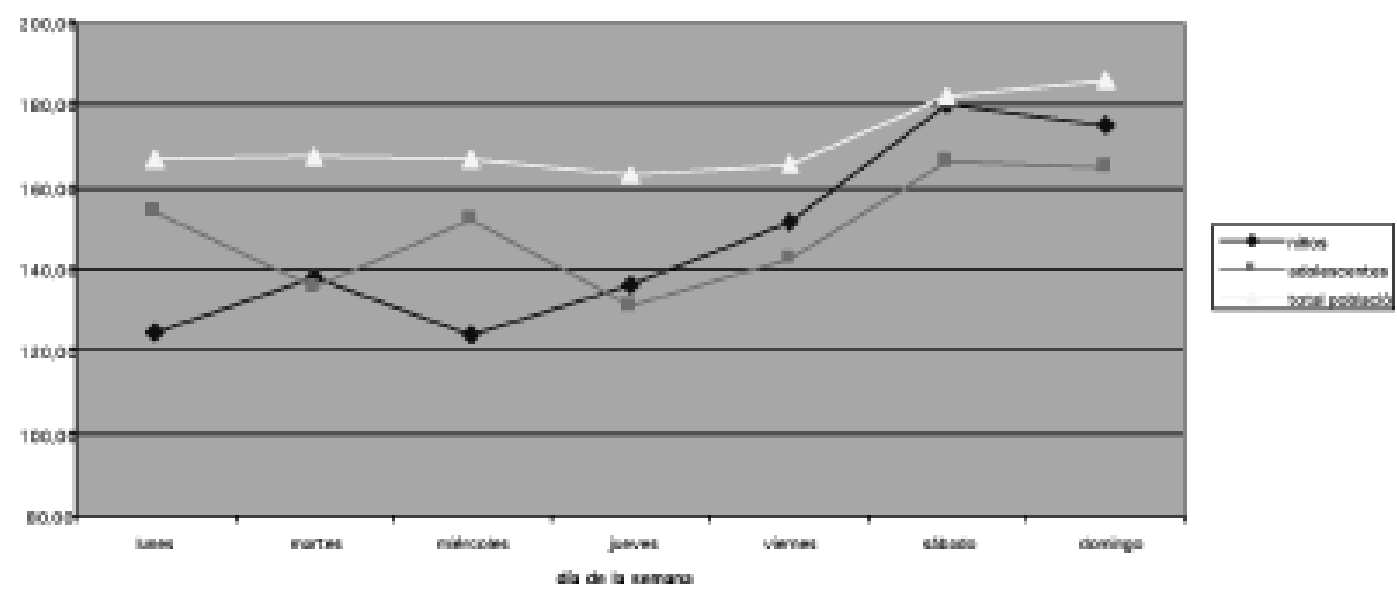

Adentrándonos descriptivamente en las diferencias de género, se observa que entre los niños (10-13 años), son las mujeres las que más televisión ven: 152,43 minutos/día de media (como actividad principal y secundaria). La media de los varones es de 146,65 minutos/día. Una diferencia por género que va en sentido contrario de lo que ocurre cuando se pasa de los 14 a los 17 años, ambos inclusive: 149,07 minutos/día las mujeres y 151,58 minutos/día los varones.

En el paso de la infancia a la adolescencia, aumenta ligeramente el consumo total televisivo por día del conjunto del grupo de edad (de 149,55 minutos/día a 150,27 minutos/día ${ }^{35}$ ); pero si se observa por género, la evolución es muy distinta: aumenta cinco minutos de media entre los varones y desciende más de dos minutos entre las chicas. Situación que va a empezar a relacionarse con la mayor asunción de tareas del hogar por parte de las chicas. De hecho, es la categoría de actividad en la que se da más diferencia de participación en estas edades, como se muestra en la siguiente tabla:

\footnotetext{
${ }_{35} \mathrm{Tal}$ aumento es mucho mayor en el subgrupo de edad que tiene 14 o 15 años, que en el que tiene 16 o 17 años, en el que se produce un descenso importante del consumo televisivo.
} 
Tabla número 2: Proporción de individuos, entre 14 y 17 años, que participan en las diversas categorías de actividades, según sexo

\begin{tabular}{cccccccc}
\hline Sexo & DORMIR & $\begin{array}{c}\text { Cuidados } \\
\text { personales }\end{array}$ & $\begin{array}{c}\text { Tiempo } \\
\text { trabajo }\end{array}$ & $\begin{array}{c}\text { Tiempo } \\
\text { estudios }\end{array}$ & $\begin{array}{c}\text { Hogar y } \\
\text { familia }\end{array}$ & $\begin{array}{c}\text { Ocio y } \\
\text { otras acti- trayectos } \\
\text { vidades }\end{array}$ & Tiempo \\
\hline Varones & $100 \%$ & $100 \%$ & $7 \%$ & $56 \%$ & $57 \%$ & $99 \%$ & $88 \%$ \\
Mujeres & $100 \%$ & $100 \%$ & $3 \%$ & $64 \%$ & $77 \%$ & $99 \%$ & $88 \%$ \\
\hline
\end{tabular}

Vemos cómo mientras sólo el 57\% de los varones de estas edades participa en alguna actividad del hogar y la familia, incluyendo el trabajo doméstico, tal proporción asciende al 77\% entre las chicas. Pero, además, la dedicación a estas tareas entre quienes participan en las mismas es muy distinta entre chicos y chicas. Los primeros le dedican algo más de una hora de media (68,97 minutos/día); mientras que las chicas sobrepasan la hora y media (100,16 minutos/día). Parece que, en principio, una mayor dedicación a las tareas domésticas hace que las chicas vean menos televisión.

Volviendo a las edades que constituyen el centro de este trabajo, entre los 10 y los 13 años, y la distribución de medias de tiempo consumido ante la televisión por Comunidades Autónomas, donde más tiempo se ve la televisión por los más pequeños es en La Rioja y Ceuta y Melilla, sobrepasando las tres horas por día, aun cuando hay que tener en cuenta el efecto estadístico de la escasa muestra para esta región y estas ciudades autonómicas. Donde menos tiempo se ve, entre los niños que la ven, es en la Comunidad de Murcia (116,54 minutos/día), la Comunidad Valenciana (124,67 minutos/día), Cantabria (130 minutos), la Comunidad de Madrid (135 minutos) y la Comunidad de Navarra $(135,17)$.

Además de la extensión del tiempo, ha preocupado a la sociedad la concentración del mismo a lo largo de determinados períodos del día, especialmente por la noche. Pues bien, hay que subrayar que a las once de la noche de un día medio laborable -entre lunes y jueves- aún permanece ante la pantalla el 15,5\% de los consultados. Tal proporción de niños irá descendiendo según avanza la noche, como muestra el siguiente gráfico: 


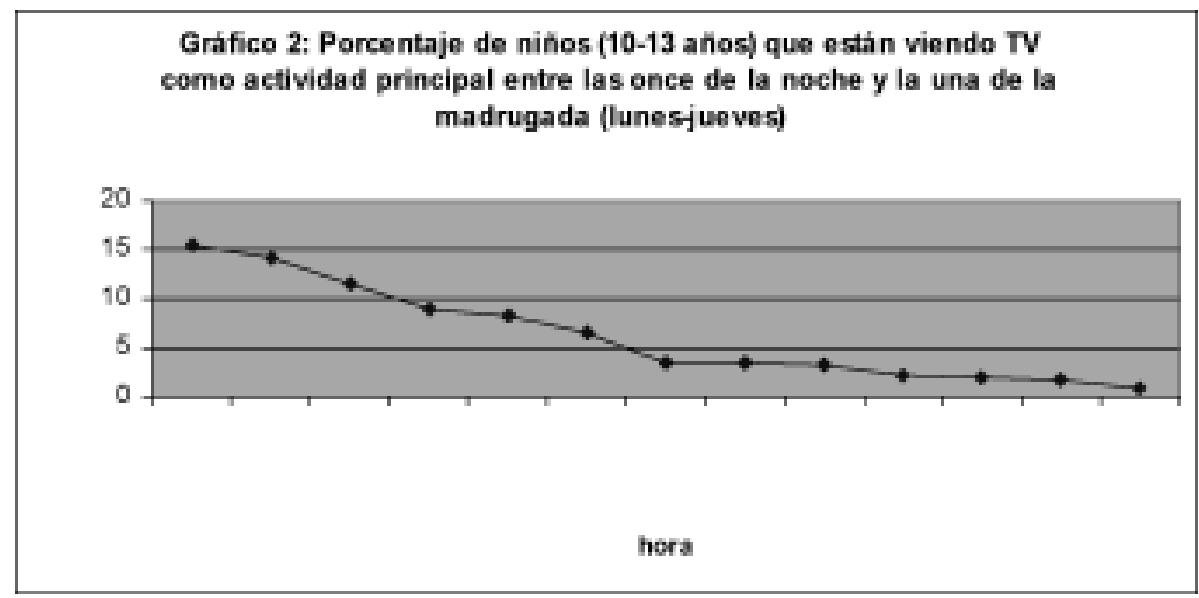

\subsection{La relación entre actividades}

Tras el subapartado anterior, que adquiere la función de marco de la relación temporal infantil con la televisión, nos introducimos en el análisis de algunos de los aspectos de ese tiempo. En primer lugar, en la relación del mismo con el tiempo destinado a otras actividades.

En primer lugar, hay que señalar que, la durante la mayor parte del día, la actividad de estudio es la principal de la jornada infantil. Así, el porcentaje de niños que realiza alguna actividad relacionada con los estudios (estudiar, ir a clase, hacer deberes) está por encima del porcentaje de niños que ve la televisión durante casi toda la jornada. La única excepción está entre las dos y media y las tres de la tarde y, ya después, a partir de las nueve de la noche. 
Gráfico 3: Porcentaje de niños que están viendo la televisión o están estudiando, como actividad principal, en un día medio

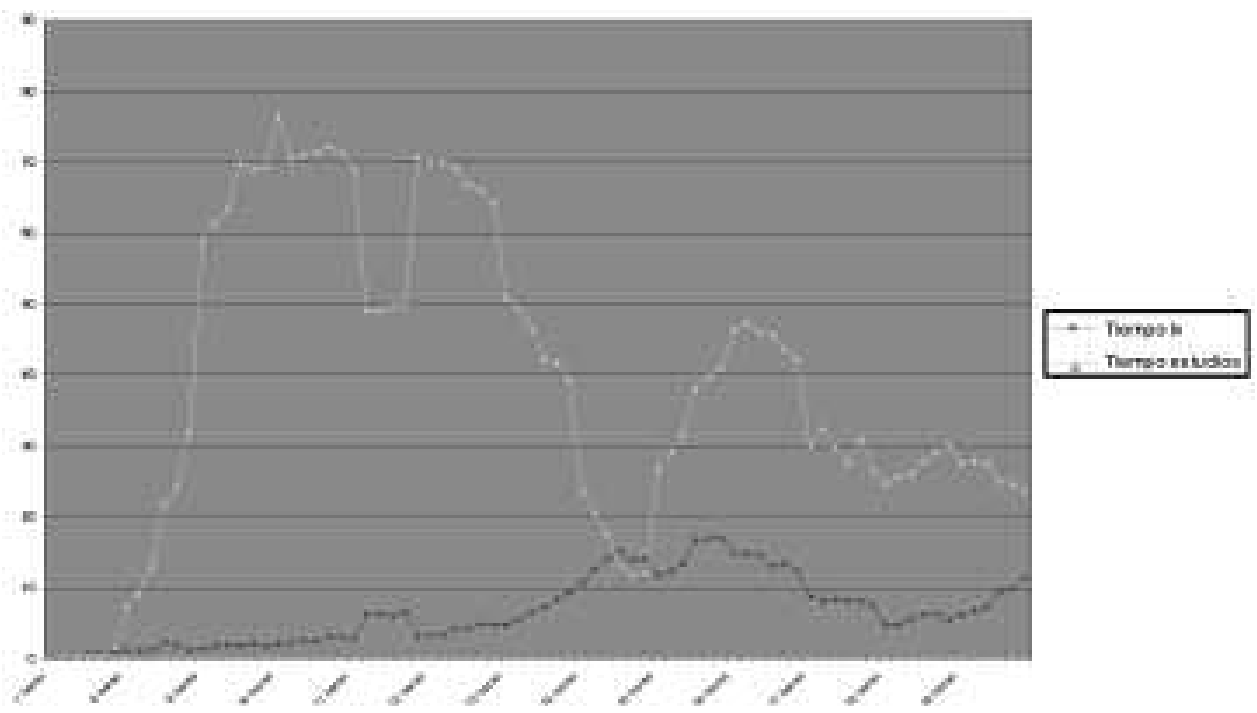

En cualquier caso, hay que subrayar que se observa una correlación negativa entre el tiempo de ver la televisión y el tiempo de estudios. No hay correlación significativa -ni positiva, ni negativa- con relación a dormir u otras actividades. Ahora bien, lo que más quita el sueño, la actividad que en mayor medida correlaciona negativamente con dormir son, con $-0,501^{36}$, son los propios estudios. De hecho, el tiempo de estudios es el que en mayor medida correlaciona negativamente con otras actividades, como cuidados personales o actividades domésticas, de manera que puede interpretarse como un obstáculo para su realización. Es más, impide más estas actividades que el propio seguimiento de televisión.

¿A qué actividades quita tiempo el consumo televisivo o qué actividades quitan tiempo a la televisión? El tiempo de estudios, como se ha señalado (correlación negativa de -0,338) y el tiempo de trayectos $(-0,209)$ correlacionan negativamente con el tiempo de estudios. Como

${ }^{36}$ Todas las correlaciones de Pearson referidas son significativas al nivel 0,01 (bilateral). 
puede apreciarse para el conjunto de los niños observados, se trata de correlaciones relativamente débiles en las que cabe intuir alguna relación causal: a más tiempo delante de la televisión, menos tiempo dedicado a los estudios; y a más tiempo dedicado a los trayectos, menos tiempo queda libre para la televisión. No obstante, en la primera relación (televisiónestudios), podría invertirse el sentido causal: más tiempo de dedicación a los estudios, deja menos tiempo para el consumo televisivo.

La idea de que la articulación de los tiempos dedicado a las distintas actividades ofrece un estilo de vida, tiene una interesante manifestación cuando se relaciona el tiempo de televisión con el tiempo de las distintas comidas ${ }^{37}$. Para su observación, se han distinguido cuatro tipos de comensales según que el tiempo dedicado a las tres comidas (desayuno, comida y cena) se encuentre por encima, alrededor o por debajo de la media. Quienes realizan las tres comidas de una manera rápida, normal o pausada conforman un tipo cada una. Un cuarto tipo está formado por quienes realizan las comidas principales (comida y cena) en un tiempo que puede considerarse normal (alrededor de la media), pero que dedican al desayuno un tiempo bastante por encima de la media, es al tipo que hemos denominado "desayuno lento". Pues bien, es este cuarto tipo de relación con la alimentación el que más tiempo dedica a la televisión; mientras que quienes llevan a cabo sus ingestas habituales de manera pausada son los que menos tiempo dedican diariamente a la televisión:

Tabla número 3: Tiempo medio de consumo televisivo infantil por estilo tiempo comida (minutos/día)

\begin{tabular}{lc}
\hline Rápido & 137,17 \\
Normal & 147,59 \\
Pausado & 135,69 \\
Desayuno lento & 160,71 \\
\hline
\end{tabular}

${ }^{37}$ La relación entre el consumo alimentario y sus desórdenes y, por otro lado, el consumo televisivo es un tema de creciente interés en la investigación. Véase, yendo más allá de la directa relación con la publicidad específica de alimentos, las investigaciones de: HARRISON, K., CANTOR, J., "The relationship between media consumption and eating disorders”, Journal of Communication, nº. 47, 1997, pp. 40-67; y GORDON, R. A., Anorexia and bulimia: Anatomy of social epidemy, Cambridge, Basil Blackwell, 1990. 
Los niños que presentan un estilo de tiempo de comida de desayuno lento son los que más tiempo dedican a la televisión, especialmente mientras desayunan.

A pesar de que en el momento en que se realiza el trabajo de campo del Estudio de Empleo del Tiempo -entre octubre 2002 y septiembre 2003- aún la proporción de hogares con acceso a Internet en España era bastante inferior a la existente en la actualidad, aparecen algunos resultados que pueden ser de interés. En primer lugar, destaca que el consumo de ordenador o Internet es relativamente escaso en estas edades. Sólo el 4,6\% ha gastado tiempo en alguna de estas actividades (estar con el ordenador o con internet). No ocurre lo mismo con las videoconsolas (Nintendo, Play, etc.), donde el 21,9\% de los niños consume algo de tiempo. Las correlaciones entre estos tiempos de consumo mediático y el consumo de televisión no han resultado estadísticamente significativas, aun cuando se aprecia una covariación negativa entre tiempo de consumo televisivo total y tiempo de estar con el ordenador o en Internet y covariación positiva entre el tiempo de consumo televisivo y el de videoconsolas ${ }^{38}$.

\subsection{Seguimiento acompañado}

En un día medio del total de la semana, el porcentaje de niños (10-13 años) que ven la televisión (como actividad principal) solos, entre la 1 de la tarde y la 1 de la mañana, no supera el 2,9\% del total de niños (entre las 13:30 y 13:40) o el 2,8\% del total de niños (entre las 14:50 y las 15:00). Entre los niños más pequeños (10-11), los que ven la televisión (como actividad principal) solos, entre la 1 de la tarde y la 1 de la mañana, los máximos porcentajes son un poco mayores en los mismos períodos: el 3,9\% entre las 13:30 y 13:40.

\footnotetext{
${ }^{38}$ El género marca bastante en el uso de estas nuevas tecnologías del entretenimiento y el juego infantil: 97,21 minutos/día y 95,17 minutos/día de tiempo de consumo digital (ordenador o Internet) y de videoconsolas respectivamente para los varones, frente a 62,84 minutos/día y 60,95 minutos/día entre las mujeres, en ambos casos cómputos entre los que han llevado las referidas actividades. También las diferencias entre edades son marcadas. Aumenta significativamente la proporción de niños que pasan su tiempo en el ordenador e Internet con el paso de los 10-11 años a los 12-13 años, lo que no ocurre con las videoconsolas; aunque puede que se esté adelantando. Del 2,2\% pasan al 6,9\% la proporción media de niños que pasa algún tiempo con el ordenador o Internet con el paso de los 11 años a los 12 años. Pero, además, los que lo usan, pasan de una media de 56,50 minutos/día a 89,50 minutos/día. El tiempo con la videoconsola también aumenta, pero muy ligeramente, en unos cinco minutos.
} 
Ahora bien, si en lugar del total de niños y teniendo en cuenta que una parte importante de los mismos se encuentra en centros escolares durante una buena parte del día, se observa solamente los que consumen televisión, la apreciación puede experimentar cierto cambio. El porcentaje, entre el total de niños que están viendo televisión como actividad principal, alcanza el 29,9\% entre la 13:00 y 13:10. Cuando se tiene en cuenta sólo a los niños con 10 y 11 años que están viendo la televisión, como actividad principal, el porcentaje asciende al 36,8\% entre las 13:20 y las 13:40. Es decir:

- $\quad$ Es la sobremesa o el tiempo antes de comer en el que los niños en mayor proporción ven la televisión solos. Algo que tal vez tenga que ver con las diferencias de horarios entre la escuela de niños y los de los padres.

- $\quad$ Los niños más pequeños (10-11 años) ven en mayor medida la televisión solos. Cuestión que podría estar indicando una tendencia a dejar a los niños solos antes delante de la televisión.

Siguiendo con estos niños más pequeños y teniendo en cuenta ahora las características del día, se tiene que las puntas de mayor porcentaje de niños que están viendo solos la televisión, entre los que llevan a cabo esta actividad como principal, están en:

- $\quad$ Los viernes entre las 13:00 y las 13:10, donde llegan al 50\%. Prácticamente entre la una de la tarde y las tres se encuentra constantemente por encima del $40 \%$.

- $\quad$ Los laborables (lunes-jueves) entre las 13:50 y las 14:00, con 24,5\%

- $\quad$ Los laborables (lunes-jueves) entre las 18:20 y las 18:30, con 24\%

De manera general, los días del fin de semana es menor la proporción de niños de estas edades menores (en los mismos tramos horarios) que están viendo solos la televisión.

\subsection{Los más consumidores}

Hasta ahora, el análisis de los resultados obtenidos se ha centrado en el conjunto de niños. Opción que ofrece una visión general; pero que impide enfocar a aquéllos segmentos de población que presentan las conductas observadas más acentuadas. En este caso, el de los 
niños más consumidores de televisión. Para ello y teniendo en cuenta las referidas importantes diferencias de consumo televisivo entre la semana laboral y el fin de semana, se ha partido de las medias del total de niños entre 10 y 13 años durante la semana laborable de lunes a jueves (130,82 minutos/día), el fin de semana (177,83 minutos/día) y el viernes (151,54 minutos/día). Pues bien, se ha identificado el grupo de niños ${ }^{39}$ cuyo consumo total de televisión está 0,5 desviaciones típicas por encima de la media, lo que lleva a situar los respectivos umbrales mínimos en: 174,18 minutos/día para los días laborables, 232,24 minutos/día en el fin de semana y 199,20 minutos/día durante el viernes. Es decir, los fuertes consumidores identificados ven casi cuatro horas de televisión durante cualquiera de los días del fin de semana, más de tres horas durante el viernes o casi tres horas durante un día cualquiera entre lunes y jueves. Veamos algunas de las diferencias entre los consumidores medios y estos fuertes consumidores, en los tres tipos de jornada semanal establecidos.

En un día laborable, la mayor diferencia entre unos y otros está en el tiempo de estudios: los que ven mucha televisión, dedican 92 minutos menos, de media, a los estudios diariamente. También se observan diferencias en los tiempos dedicados a dormir y a trayectos; pero son de menor cuantía absoluta, a pesar de que el tiempo de dormir es bastante extenso en sí mismo. Para evitar la desfiguración en la comparación que puede derivar de la extensión absoluta que suelen llegar las distintas categorías de actividad, se hace necesario relativizarlas, siendo conveniente referir cada tiempo de cada categoría de actividad a la otra, según las diferencias porcentuales. Así se obtiene que los niños que ven mucha televisión dedican un 22\%, un 20\% y un $16 \%$ menos a los estudios respectivamente durante los días laborables, los viernes y los días del fin de semana, que el resto de los niños.

También como se apuntaba antes, la otra categoría de actividades que aparece afectada por el tiempo televisivo son los trayectos: los niños que ven mucha televisión dedican un 27\% (laborables), 22,4\% (viernes) y 32,4\% (fin de semana) menos de tiempo a los trayectos. Aun cuando cabría señalar que tiene más sentido hablar de que es el tiempo televisivo el que se ve

\footnotetext{
${ }^{39} \mathrm{Al}$ utilizar la desviación típica como umbral de referencia, el porcentaje de los niños que aparecen viendo mucha televisión es semejante en los tres tipos de día de la semana: alrededor de la cuarta parte de los niños (ya que se ha utilizado el 0,5 de desviación típica por encima de la media).
} 
afectado, con una reducción del mismo, por el tiempo dedicado a los viajes y trayectos, especialmente en los días del fin de semana.

La mayor dedicación a las tareas de hogar y familia por parte de los niños que ven mucha televisión, con respecto al resto de niños, cabe explicarla porque entre los primeros hay un mayor porcentaje de chicas durante los tres tipos de jornada semanal.

Durante la semana laboral, incluyendo el viernes, el fuerte seguimiento televisivo no sólo no afecta al tiempo de sueño o de cuidados personales -en los que está incluido el propio dormirsino que los que ven mucha televisión duermen un poco más. Situación que se invierte durante los días del fin de semana, de manera que parece que el tiempo televisivo roba algo de tiempo al sueño.

Visto conjuntamente la manera en que el mucho tiempo de televisión infantil afecta al tiempo de las otras categorías, podría decirse que los niños y, sobre todo, niñas que consumen mucho tiempo televisivo dedican buena parte del día a estar en casa, pues, salvo en el caso del tiempo de sueño y cuidados personales durante el fin de semana, tienden a dedicar más tiempo a las categorías de actividades que tienden a realizarse preferentemente en el ámbito doméstico. Se trata de niños y niñas más domésticos.

Tabla número 4: Diferencia porcentual en las diferentes categorías de actividades, entre los niños que ven mucha televisión y el resto de niños, durante los tres tipos de días de la semana

\begin{tabular}{lccccc}
\hline Actividad & Dormir & $\begin{array}{c}\text { Cuidados } \\
\text { personales }\end{array}$ & Estudios & Hogar y familia & $\begin{array}{c}\text { Tiempo de } \\
\text { trayectos }\end{array}$ \\
\hline Laborable & 1,9 & 0,1 & -22 & 19,9 & $-27,0$ \\
Viernes & 3,0 & 2,8 & -20 & 28,4 & $-22,4$ \\
Fin semana & $-1,4$ & $-1,9$ & -16 & 10,5 & $-32,4$ \\
\hline
\end{tabular}




\section{Conclusiones}

La observación en la población infantil de la articulación del consumo televisivo con el tiempo dedicado a otras actividades, a través de la Encuesta de Empleo del Tiempo, ha producido las siguientes conclusiones:

a) Una aproximación a la relación con un medio de comunicación centrada en la dimensión tiempo nos acerca bastante al valor que la sociedad o un grupo determinado de la misma, como en este caso los niños, da a tal relación en sus vidas. Más cuando tal tiempo de consumo se concibe como un recurso limitado, 24 horas al día, del que hay que disponer para distintas actividades. Pero, además, ofrece un buen retrato de la vida de esa sociedad o de ese grupo social en particular, poniendo el medio de comunicación como eje de tal retrato, aun cuando el mismo refleje situaciones como: la de que casi uno de cada seis niños entre 10 y 13 años estén delante del televisor a las once de la noche en la víspera de un día de escuela; o la de que alrededor de una cuarta parte de estos niños, los identificados como fuertes consumidores de televisión, se sitúa alrededor de las tres horas de consumo televisivo de media durante cualquiera de los días laborables y de las cuatro horas durante un día del fin de semana.

b) La media de consumo televisivo infantil se sitúa alrededor de las dos horas y media, siendo uno de los grupos de edad que menos tiempo dedica a la televisión en el territorio español, debiéndose tener en cuenta que la media de la población general se sitúa cerca de las tres horas diarias. Por otro lado y como cabía esperar, el consumo medio televisivo infantil experimenta importantes variaciones a lo largo de la semana, siendo mayor durante los sábados.

c) El paso de la niñez a la adolescencia implica algunos cambios en la relación con la televisión. Por un lado, aumenta ligeramente en el inicio de la adolescencia (14-15 años), para descender en los siguientes pasos (16-17 años). Por otro lado, si las niñas (10-13 años) ven más televisión que los niños, los adolescentes (14-17 años) ven más televisión que las adolescentes. La explicación a esta última variación se ha encontrado en el distinto grado de implicación en las tareas domésticas por parte de varones y mujeres. 
d) Los resultados permiten matizar algunas de las conclusiones obtenidas en otros países, en especial, las que relativizan la influencia de la cantidad de tiempo de consumo televisivo infantil sobre otras actividades ${ }^{40}$. Al menos se ha puesto de relieve aquí la relación existente entre tiempo de estudio y tiempo de consumo televisivo, estando en correlación negativa. Una relación negativa que aumenta considerablemente en el caso de los que han sido identificados como fuertes consumidores de televisión, llegando a dedicar entre un $16 \%$ y un $22 \%$ menos de tiempo a los estudios que el resto de niños, según el tipo de día de la semana de que se trate. Sin embargo, hay que matizar que durante la mayor parte del día la proporción de niños que realiza actividades vinculadas con los estudios y el aprendizaje es mayor que la proporción de niños que está consumiendo televisión.

e) Identificado el grupo de fuertes consumidores de televisión, además de menos dedicado a los estudios que el resto de niños, comparativamente se ha perfilado como un grupo de niños bastante centrados en el hogar, en función de las actividades con las que se articula ese mayor consumo televisivo.

f) Por último, hay que destacar que el análisis del material empírico no sólo ha puesto de relieve lo que pueden considerarse consecuencias negativas del tiempo televisivo. Por un lado, parece facilitar que algunas comidas se realicen a un ritmo más pausado, como ocurre con el desayuno. Por otro lado y si se tiene en cuenta el total de niños, es relativamente poco el porcentaje (alrededor del 3\% y 4\%, según la edad y la hora) de los mismos que consumen solitariamente el medio. Ahora bien, si se tienen en cuenta sólo los niños que están viendo televisión en cada momento, los porcentajes de seguimiento solitario pueden dispararse, hasta casi el $50 \%$ en algún período de la semana (salida matinal del colegio del viernes). Es decir, en algunos momentos del día y la semana, no se encuentra mejor ubicación para los niños que llegan a casa, frente a los que se quedan a comer en la escuela, que delante de la pantalla.

${ }^{39}$ Cfr. MUTZ y otros, op. cit. 


\section{Referencias bibliográficas}

ALONSO, M., MATILLA, L., VÁZQUEZ, M., Los teleniños, Barcelona, Laia, 1986.

ALONSO, M., MATILLA, L., VÁZQUEZ, M., Teleniños públicos, teleniños privados, Madrid, Quirón, 1995.

BOURDIEU, P., El sentido práctico, Madrid, Taurus, 1991.

BURTON, J., CALONICO, J., MCSUENY, D., “Growing up with televisión: Effects of preschool televisión watching on first-grade children”, Journal of Communication, nº. 29, 1979.

CALLEJO, J., "Limitaciones para el establecimiento de una tipología de la investigación sobre niños y televisión: el caso español”, en Empiria. Revista de Metodología de Ciencias Sociales, no. 8, julio-diciembre 2004.

CALLEJO, J., Investigar las audiencias, Barcelona, Paidós, 2001.

CAMPBELL, D., y STANLEY, J., Diseños experimentales y cuasiexperimentales en la investigación social, Buenos Aires, Amorrortu, 1991.

CORBETTA, P., Metodología y técnicas de investigación social, Madrid, McGraw-Hill, 2003.

DURÁN, M. A., La cuenta satélite del trabajo no remunerado en la Comunidad de Madrid, Madrid, Comunidad de Madrid, 2006.

FARNÉ, R., "Il problema della recerca su bambini e televisione", ponencia del I Congreso Internacional la Televisión y la Infancia, Madrid, 17-19 de octubre, 2007.

FERRÉS, J., Educar en una cultura del espectáculo, Barcelona, Paidós, Barcelona, 2003.

GARCÍA GALERA, M. C., Televisión, violencia e infancia. El impacto de los medios, Barcelona, Gedisa, 2000.

GARCÍA MATILLA, A., Una televisión para la educación. La utopía posible, Barcelona, Gedisa, 2003. 
GARCÍA MATILLA, A., CALLEJO GALLEGO, J., y WALZER, A., Los niños y los jóvenes frente a las pantallas, Madrid, Ministerio de Trabajo y Asuntos Sociales, 2004.

GORDON, R. A., Anorexia and bulimia: Anatomy of social epidemy, Cambridge, Basil Blackwell, 1990.

HARRISON, K., CANTOR, J., "The relationship between media consumption and eating disorders”, Journal of Communication, nº. 47, 1997.

HIMMELWEIT, H., OPPENHEIM, A., y VINCE, P., Television and the child: An empricial study of the effects of television on the young, Londres, Oxford University Press, 1958.

LEÓN, J. M., CANTERO, F. J., GÓMEZ, T., “Efectos de la clasificación del comportamiento del modelo simbólico televisado sobre la agresividad infantil”, Revista de Psicología Social, 12 (1), 1997.

LIVINGSTONE, S., "The camping nature of audiences: from the Mass Audience to the interactive media user", en Valdivia, A (ed.), The Blacwell Companion to Media Research, Blackwell, Oxford, 2003.

LIVINGSTONE, S., y BOBER, M., UK Children Go Online: Final Report of Key Project Findings, London School of Economics and Political Science, Londres, 2005.

LLOPIS GOIG, R., "La mediación familiar del consumo infantil de televisión. Un análisis referido a la sociedad española”, Comunicación y Sociedad, vol XVII (2), 2004.

LUHMANN, N., Observaciones de la modernidad. Racionalidad y contingencia en la sociedad moderna, Barcelona, Paidós, 1997.

LUHMANN, N., La Ciencia de la Sociedad, México, Anthropos, 1996.

MARTA LAZO, C., La televisión en la mirada de los niños, Madrid, Fragua, 2005.

MUTZ, D. C., ROBERTS, D. F., VAN VUUREN, D. P., "Reconsidering the displacement hypothesis: Television's influence on children's time use”, Communication Research, nº.20, 1993. 
NÚÑEZ LADEVĖZE, L., y TORRECILLA LACAVE, L., “El contexto de la recepción infantil”, en Telos, nº. 73, octubre-diciembre 2007.

NÚÑEZ LADEVĖZE, L., PÉREZ ORNIA, J. R. "Programación infantil en la televisión española. Inadecuada relación entre oferta y demanda”, Telos, nº. 54, enero-marzo, 2003.

NÚÑEZ LADEVĖZE, L., y PÉREZ ORNIA, J. R., "Los gustos de la audiencia infantil y la producción televisiva. El conflicto pragmático de los responsables de la audiencia infantil”, en Revista Española de Investigaciones Sociológicas, 99, 2002.

PEÑA, M. E., ANDREU, J. M., MUÑOZ, M. J., "Efectos de la visión de escenas violentas en la conducta agresiva infantil, Psicothema, vol. 11 (1), 1999.

PÉREZ TORNERO, J. M., El desafío educativo de la televisión. Para comprender y usar el medio, Barcelona, Paidós, 1994.

PRIETO, C., y RAMOS, R., "El tiempo de trabajo entre la competitividad y los tiempos sociales”, en Las relaciones de empleo en España, F. Miguélez y Carlos Prieto (coords). Madrid, Siglo XXI, 1999.

RAMOS, R., Cronos dividido, Madrid, Centro de Investigaciones Sociológicas, 1990.

RODRÍGUEZ MÉNDEZ, J. M., Los teleadictos, Barcelona, Laia, 1973.

RODRÍGUEZ PASCUAL, I., "Redefiniendo el trabajo metodológico cualitativo con niños: el uso de la entrevista de grupo aplicada al estudio de la tecnología”, en Empiria. Revista de Metodología de Ciencias Sociales, nº. 12, julio-diciembre 2006.

SÁNCHEZ HORCAJO, J. J., “Televisión y familia: Comunicación familiar, pasividad y rendimiento escolar”, en Sociedad y Utopía, nº.1 y 2, 1993.

SCHRAMM, W., LYLE, J., y PARKER, E. B., Televisión para los niños, Barcelona, Hispano Europea, 1965.

SZALAI, A. (ed.), The use of time, The Hague, Mouton, 1972.

VALLEJO-NÁJERA, A., Mi hijo ya no juega, sólo ve la televisión, Madrid, Temas de Hoy, 1987. 
VERA, M. J., “¿Cómo perciben los niños la televisión?”, en AA. VV., Televisión, niños y jóvenes, Valencia, RTVV, 1994.

VILCHEZ MARTIN, L. F., Televisión y familia, Un reto educativo, Madrid, PPC, 1999.

WEBER, M., Economía y Sociedad, México, Fondo de Cultura Económica, 1979.

YOUNIS HERNÁNDEZ, J. A., El aula fuera del aula. La educación invisible de la cultura audiovisual, Las Palmas de G. C., Nogal, 1993.

YOUNIS HERNÁNDEZ, J. A., El niño y la cultura audiovisual, Santa Cruz de Tenerife, Centro de la Cultura Popular Canaria, 1988. 